\title{
Multeity of Magnetic Resonance and Its Applications
}

\author{
Vladimir I. Chizhik ${ }^{1}$ Murat S. Tagirov ${ }^{2}$
}

Published online: 14 March 2019

๑) Springer-Verlag GmbH Austria, part of Springer Nature 2019

In modern physics, the term "magnetic resonance" refers to a set of phenomena accompanied with the emission or absorption of electromagnetic waves of the radiofrequency diapason by quantum systems (nuclei, electrons, atoms, molecules, etc.). These phenomena, the physical nature of which is of independent interest, provide the basis of radiospectroscopic methods for studying the structure of matter and physico-chemical processes in it. They are also used for different practical applications including the creation of quantum generators, amplifiers, and magnetometers. For the development of ideas and applications of magnetic resonance six Nobel Prizes were awarded in the areas of physics, chemistry, biology, physiology and medicine (the latter was in 2003). Primarily, the magnetic resonance methods are: Nuclear Magnetic Resonance (NMR), Electron Paramagnetic Resonance (EPR), Nuclear Quadrupole Resonance (NQR), various implementations of double (and multiple) resonances. These methods allow the investigations of media in any aggregate state. Being contactless, they do not destroy an object under a study, that makes them unique and in demand not only in physics and chemistry, but also in medicine, geology, biology, archeology. Now, any medical center with high reputation has a magnetic resonance imaging (MRI). We hope that this special issue to some extent demonstrates the multeity of magnetic resonance and its applications.

In the science of the twenty-first century, great importance is attached to the problems of biology and medicine. The issue presents a few articles in these areas. In the work of T. Titma, Min-Ji Shin, C. Ludwig, et al. the metabolic profile of filtered serum of patients with the ischemic heart disease (IHD) using proton nuclear magnetic resonance spectroscopy is evaluated, namely, the filtered venous serum from age, gender matched IHD patients and control individuals were analyzed. NMR chemometrics showed a significant distinction between patients and control individuals. The principal component analysis of ${ }^{1} \mathrm{H}$ NMR detected serum metabolites exhibit

Vladimir I. Chizhik

v.chizhik@spbu.ru

Murat S. Tagirov

Murat.Tagirov@kpfu.ru

1 Saint Petersburg State University, Saint Petersburg, Russia

2 Kazan Federal University, Kazan, Russia 
a significant difference of IHD patients and control individuals. These data demonstrate that metabolomics approach may be useful for the early detection of IHD, for the detection of synergistic pathways involved in the development of altered health conditions, and for molecular understanding of particular health condition.

Authors of the article "MR relaxation studies of hemoglobin aggregation process in sickle cell disease. Application for diagnostics and therapeutics" (M. A. Lores Guevara, J. C. García Naranjo, and C. A. Cabal Mirabal) summarized the contribution of the method of proton magnetic relaxation in the study of a serious blood disorder causing acute pain, severe anemia, infections, and vascular blockages that can lead to widespread organ damage and death. They have obtained remarkable information about the behavior of protein and water protons as a consequence of hemoglobin $\mathrm{S}(\mathrm{HbS})$ polymerization. Spin-lattice $\left(T_{1}\right)$ and spin-spin $\left(T_{2}\right)$ relaxation times are sensitive to the $\mathrm{HbS}$ agglutination. The $T_{2}$ behavior shows the possibility and utility for the evaluation of clinical states of SCD patients, as well as, of the effect of vanillin and hydroxyurea on the $\mathrm{HbS}$ polymerization.

An international scientific team presents the article "Spin-lattice relaxation and diffusion processes in aqueous solutions of gadolinium-based upconverting nanoparticles at different magnetic fields" (K. Kristinaityte, T. Zalewski, Marek Kempka, et al.). Using the relationship between NMR relaxation and diffusion characteristics the authors could estimate not only the total impact of Gd-based upconverting nanoparticles (NPs) on the relaxation of water protons but also the impact on the ${ }^{1} \mathrm{H}$ relaxation of water, directly connected to paramagnetic $\mathrm{Gd}^{3+}$ ions in NPs. They supposed that the NPs investigated could be very promising as contrast agents in MRI over a broad range of magnetic fields used in clinical routine.

Increasing the sensitivity during the registration of complex NMR spectra is an important problem, especially in the case of NMR spectroscopy of biological molecules. A new approach to improve the registration of weak signals for the particular fragments of NMR spectra is proposed in the article "Multiplet-matched filtering of ${ }^{103} \mathrm{Rh}$ signal using information contained in ${ }^{31} \mathrm{P}$ spectrum of $\mathrm{AA}$ ' $\mathrm{XX}$ ' ${ }^{31} \mathrm{P}-{ }^{103} \mathrm{Rh}$ Spin System" of I. S. Podkorytov, T. G. Cherkasova, E. A. Kayfadzhyan, and Yu. S. Varshavsky. The authors present the application of a matched filter for the concrete example of a spectrum (doublet of doublets) when the splitting and line width are known. Similar multiplet-matched filters can be applicable in one- and two-dimensional NMR spectroscopy if a spectral pattern is known from other experiments or theoretical calculations.

NMR spectroscopy of paramagnetic liquid systems is a special direction in which the degree of influence of paramagnetic components both on the positions of spectral lines (chemical shifts) and on the intensity of relaxation processes plays an important role. It is worth noting that the majority of NMR studies of paramagnetic compounds is related to lanthanides complexes which are characterized by short times of electronic relaxation of rare-earth ions. Therefore, the complex formation, as a rule, leads to insignificant broadening of resonance signals as compared to paramagnetic chemical shifts. That is why these species are used as (lanthanide) shifting reagents. The article "NMR spectra of 1-vinylimidazole, transformed by paramagnetic complexes" of V. K. Voronov, I. A. Ushakov, and E. A. Funtikova is devoted to paramagnetic complexes of manganese, cobalt, nickel and copper chlorides (the first 
transition group). The authors paid special attention to fine details of the molecular structure and dynamics of azole derivatives (on the example of 1-substituted imidazole). The five-membered heterocyclic compounds, containing two nitrogen atoms in a cycle, are ubiquitous in the nature and play an important role in biological processes and find widespread application in medicine and engineering.

Two articles reflect solid matter studies. In the work "Influence of morphology of $\mathrm{LaF}_{3}$ nanocrystals on fluorine dynamics studied by NMR diffusometry" (L. B. Gulina, M. Schikora, A. F. Privalov, et al.) the ionic dynamics at the surface of nanostructured 2D superionic conductors $\mathrm{LaF}_{3}$ is analyzed on the basis of data of the ${ }^{19} \mathrm{~F}$ NMR Static Field Gradient (SFG) diffusometry in a temperature range up to $800 \mathrm{~K}$. It was necessary to measure diffusion coefficients of the order $10^{-12} \div 10^{-13} \mathrm{~m}^{2} / \mathrm{s}$. Fluorine diffusion in studied materials is about $2 \div 3$ orders of magnitude faster than in bulk $\mathrm{LaF}_{3}$. It was shown that diffusion coefficients and activation energies depend on the thickness of nano-sheets.

The development of industry occurs as a result of the emergence of new chemical technological processes. To a large extent, progress is facilitated by the widespread use of catalysts, since they help to transform raw materials into high-grade products, plastics, chemicals, drugs, fuels, fertilizers and other utility products. It determines the relevance of the work of I. V. Yakovlev, E. Papulovskiy, E. A. Paukshtis, et al. "1 $\mathrm{H}$ and ${ }^{93} \mathrm{Nb}$ solid-state NMR and IR study of acidity of nanodisperse $\mathrm{Nb}_{2} \mathrm{O}_{5} \cdot n \mathrm{H}_{2} \mathrm{O}$ '. Niobium oxide hydrate is a promising material that can find applications in various heterogeneous catalytic processes due to its strong acidity and stability in aqueous medium. The authors successfully synthesized and characterized nano-disperse niobium oxide hydrate. Solid-state NMR spectroscopy in combination with ab initio calculations gave a satisfactory insight into the nature of strong acidic sites and proved to be a useful tool for the identification of acidic sites in $\mathrm{Nb}_{2} \mathrm{O}_{5} \cdot n \mathrm{H}_{2} \mathrm{O}$ systems.

The content of the two articles is related to some aspects of the electron paramagnetic resonance. Negatively charged nitrogen vacancy (NV) color centers in diamond crystals have been intensively studied in the last few decades using the optically detected magnetic resonance (ODMR) method which usually implies resonance excitation using a DC or pulsed drive field in the gigahertz range (MW field). Various techniques for multi-frequency MW excitation of magnetic resonance in the structures containing the ${ }^{14} \mathrm{~N}$ atoms (NV centers) were developed. Most of them use the MW excitation in combination with RF modulation or direct RF excitation of nuclear transition. In the article "Ultra-narrow low-field nuclear spin resonance in NV centers in a bulk diamond crystal" A. K. Dmitriev and A. K. Vershovskii report on the possibility to detect ultra-narrow resonances in the nuclear structure of ${ }^{14} \mathrm{~N}$ using the ODMR methods with single-frequency DC RF excitation of the nuclear transition at $4.95 \mathrm{MHz}$. The resonances were detected in a bulk sample in weak magnetic fields $(0 \div 10 \mathrm{mT})$. These resonances may be of interest for solidstate quantum information processing, as well as for quantum magnetometry, especially in biological and medical applications where a strong MW drive field may be undesirable.

New compact EPR equipment is described in the article "Features of development and applications of compact EPR analyzers" of Yu. V. Bogachev, M. N. 
Knyazev, and A. V. Nikitina. The equipment is developed both for research and for analytical applications. The described instrumentation can be characterized by high performance, low cost, reliability and simplicity, open architecture, small size, a wide range of functional modules and units. The authors developed and experimentally investigated two cavities of optimal construction for autodyne type EPR analyzers. Specifications of the new compact EPR equipment and a list of applications are also presented.

The last article of this special issue is devoted to nuclear quadrupole resonance (NQR): V. V. Ogloblichev, V. L. Matukhin, I. Yu. Arapova, C. V. Schmidt, R. R. Khusnutdinov ${ }^{663,65} \mathrm{Cu} \mathrm{NQR}$ spectra and spin-lattice relaxation in thermoelectric $\mathrm{CuAlO}_{2}$ ". The value of the nuclei quadrupole interaction constant $\mathrm{QCC}=56.24 \mathrm{MHz}$ (at $T=298 \mathrm{~K}$ ) for the semiconductor compound $\mathrm{CuAlO}_{2}$ has been obtained. The broad maximum has been found in the temperature dependence of $1 / T_{1}$ in low-temperature region (below $276 \mathrm{~K}$ ) that can be associated with the presence of energy levels in the forbidden band. Assuming the activation character of the mobility of holes in the semiconductor $\mathrm{CuAlO}_{2}$ it was possible to estimate an activation energy $E_{\mathrm{a}}=45 \mathrm{meV}$.

It would be satisfying if the publications presented in this issue will attract the attention of scientific community and will stimulate further progress in the magnetic resonance and its applications.

Publisher's Note Springer Nature remains neutral with regard to jurisdictional claims in published maps and institutional affiliations. 